\title{
Les produits agroalimentaires du terroir au Québec : un état des lieux du marché et du profil des consommateurs
}

\author{
Catherine Parissier, Ph. D. et Bénédicte Geay, M. Sc. \\ Université de Sherbrooke
}

\section{INTRODUCTION}

Les produits du terroir sont en effet

théoriquement des produits répondant à

des cahiers des charges stricts, mais

nombreux sont les producteurs industriels ou artisanaux qui utilisent le label

" terroir " de manière parfois abusive pour mieux commercialiser leurs produits.

Depuis plusieurs années, les produits agroalimentaires de niche (tels que les produits régionaux, artisanaux, fermiers ou traditionnels) connaissent un engouement certain au Québec. Grâce au soutien des médias traditionnels ${ }^{1}$ ou numériques $^{2}$, ces produits sont de plus en plus recherchés par les consommateurs, à la fois pour leur qualité (ils sont perçus comme plus naturels et donc meilleurs pour la santé), pour leur goût, mais également pour leur symbolisme, ces produits étant pour les individus une manière d'affirmer leur identité régionale, culturelle et sociale $^{3}$. Bien que ce marché des produits agroalimentaires de niche soit présenté généralement comme un "tout» ou un ensemble homogène, force est de constater qu'il recouvre des réalités diverses parmi lesquelles les produits du terroir offrent un exemple probant. Les produits du terroir sont en effet théoriquement des produits répondant à des cahiers des charges stricts, mais nombreux sont les producteurs industriels ou artisanaux qui utilisent le label «terroir» de manière parfois abusive pour mieux commercialiser leurs produits. La confusion qui en découle, à la fois pour les professionnels de l'agroalimentaire et pour les consommateurs, peut fortement nuire à la promotion de ces produits et de manière générale à l'économie des terroirs. Il est donc aujourd'hui essentiel de mieux définir ce que sont les produits du terroir, de mieux comprendre la réalité de ce marché (catégories de produits, profil des consommateurs) et la perception des consommateurs à leur égard pour contribuer à leur différenciation et ainsi à leur essor. Cette étude présente donc les premiers résultats d'une recherche universitaire ${ }^{4}$ ayant eu pour objectif de dresser un premier état des lieux du marché des produits agroalimentaires du terroir au Québec (catégories de produits et profil type des consommateurs). Elle s'appuie sur l'étude documentaire d'une centaine de sources d'information, puis sur une recherche qualitative basée sur des entrevues réalisées auprès de quatorze experts du secteur des produits agroalimentaires du terroir au Québec. Dans un premier temps, une définition des produits du terroir sera proposée ainsi qu'une clarification du processus de certification actuellement en cours dans la province. Dans un second temps, après que la méthodologie employée ait été clarifiée, les principaux résultats de la recherche seront exposés. Finalement, nous conclurons sur son intérêt pratique pour des recherches ultérieures visant une meilleure connaissance et promotion des produits du terroir au Québec.

Il est donc aujourd'hui essentiel de mieux définir ce que sont les produits du terroir, de mieux comprendre la réalité de ce marché (catégories de produits, profil des consommateurs) et la perception des consommateurs à leur égard pour contribuer à leur différenciation et ainsi à leur essor. 


\section{DÉFINITION DES PRODUITS DU TERROIR ET PROCESSUS DE CERTIFICATION}

\subsection{Les cinq caractéristiques des produits du terroir au Québec}

Bien que Solidarité Rurale du Québec ait recensé en $2002^{5}$ soixante-treize produits du terroir agroalimentaire, forestier, minéral ou végétal, il n'existe actuellement pas de définition consensuelle de la notion de «produit du terroir» au Québec. Selon les sources documentaires consultées ou les organismes publics et associatifs qui souhaitent établir un cadre qui les régirait, les définitions diffèrent. Toutefois, la confrontation de trois définitions émanant d'organismes publics notables (Conseil des Appellations Réservées et des Termes Valorisants $(\mathrm{CARTV})^{6}$; Groupe de travail sur les appellations réservées et les produits du terroir mandaté par le ministère de l'Agriculture, des Pêcheries et de l'Alimentation du Québec (MAPAQ); ministère des Affaires municipales, des Régions et de l'Occupation du territoire du Québec) a permis d'identifier cinq caractéristiques communes (citées dans les définitions consultées) qui illustrent le caractère distinctif des produits du terroir au Québec : la zone géographique, les facteurs naturels, les facteurs humains, la tradition et le temps, la différenciation ${ }^{7}$.

\section{Le produit du terroir est intrinsèquement issu d'une zone ou d'un territoire géographique délimité qui présente des facteurs naturels (exemples : climat, sol) et humains spécifiques (savoir-faire local tel que le mode de transformation d'un produit par exemple). De surcroît, il est élaboré selon un procédé ancré dans l'histoire des habitants dudit territoire (datant d'au moins une génération) et transmis « de père en fils ».}

Selon ces caractéristiques, le produit du terroir est intrinsèquement issu d'une zone ou d'un territoire géographique délimité qui présente des facteurs naturels (exemples: climat, sol) et humains spécifiques (savoir-faire local tel que le mode de transformation d'un produit par exemple). De surcroît, il est élaboré selon un procédé ancré dans l'histoire des habitants dudit territoire (datant d'au moins une génération) et transmis «de père en fils » (tradition). Enfin, conséquemment, la différenciation illustre le fait que le produit du terroir est unique ou particulier dans le sens où il ne pourrait être produit nulle part ailleurs et est donc non reproductible dans une autre zone géographique distincte de son origine. Pour être nommé et reconnu comme tel, un produit du terroir devrait donc cumuler ces cinq spécificités.

\subsection{La certification et la labellisation des produits du terroir au Québec}

En avril 1996, le Québec se dote d'une loi sur les appellations réservées pour permettre d'octroyer de manière discriminante une certification (les Appellations d'origine (AO) et les Indications Géographiques Protégées (IGP)) à des produits présentant des caractéristiques propres les différenciant de manière significative. Pour engager réellement la mise en place de ces dispositions (aucune appellation d'origine ou indication géographique protégée n'ayant été décernée suite à cette réglementation de 1996), une nouvelle loi est adoptée en avril 2006 portant cette fois sur les appellations réservées et les termes valorisants. Le CARTV est alors créé et a la responsabilité de veiller à la mise en place de ce processus de certification en décernant les signes officiels d'identification de la qualité et de l'origine du produit (les appellations réservées, objet de la loi) qui, au Québec, peuvent prendre quatre formes particulières: l'appellation biologique, l'attestation de spécificité, l'appellation d'origine (AO) et l'indication géographique protégée (IGP). Parmi ces quatre formes de certification, les appellations d'origine et les indications géographiques protégées adossées à un cahier des charges publiques portant sur les caractéristiques du produit et ses conditions et zones de production, sont clairement positionnées comme "relatives au lien avec un terroir », mais l'usage de la dénomination " produit du terroir » demeure non encadré d'un point de vue légal. L'examen attentif des définitions respectives des appellations d'origine et des indications 
géographiques protégées montre de plus que ces dernières recouvrent très peu (IGP) ou seulement partiellement $(\mathrm{AO})$ les cinq caractéristiques clés identifiées dans la littérature comme typiques des produits du terroir, ouvrant donc encore une fois la voie à un usage parfois abusif de la dénomination «terroir» (Tableau 1). En mars 2009, la première indication géographique protégée est décernée à l'agneau de Charlevoix, mais cette expérience demeure cependant unique. La réglementation en vigueur et son application encadrent donc actuellement peu les produits du terroir au Québec.

\section{En mars 2009, la première indication géographique protégée est décernée à l'agneau de Charlevoix, mais cette expérience demeure cependant unique. La réglementation en vigueur et son application encadrent donc actuellement peu les produits du terroir au Québec.}

Tableau 1 : Les appellations réservées au Québec relatives au lien avec le terroir

\begin{tabular}{|c|c|c|c|c|c|c|c|c|c|c|}
\hline & \multicolumn{5}{|c|}{ Appellation d'origine } & \multicolumn{5}{|c|}{ Indication géographique protégée } \\
\hline Définition & \multicolumn{5}{|c|}{$\begin{array}{l}\text { "(Elle) est la dénomination géographique } \\
\text { d'un pays, d'une région ou d'une localité } \\
\text { servant à désigner un produit qui en est } \\
\text { originaire. L'appellation d'origine consacre } \\
\text { donc les facteurs naturels d'un terroir dont } \\
\text { les caractéristiques géologiques, } \\
\text { agronomiques, climatiques, techniques } \\
\text { (conditions de production) et humaines } \\
\text { (savoir-faire et pratiques) permettant de } \\
\text { définir la spécificité et la personnalité du } \\
\text { produit. L'appellation d'origine implique } \\
\text { donc un lien très étroit entre le produit, le } \\
\text { terroir et le savoir-faire de l'homme. C'est } \\
\text { pourquoi tous les stades de réalisation du } \\
\text { produit soit sa production, son élaboration } \\
\text { et sa transformation doivent avoir lieu dans } \\
\text { la région de l'appellation " }{ }^{8} \text {. }\end{array}$} & \multicolumn{5}{|c|}{$\begin{array}{l}\text { «(Elle) établit principalement un lien entre } \\
\text { un produit et une région. Elle désigne un } \\
\text { produit dont une qualité déterminée, la } \\
\text { réputation ou une autre caractéristique peut } \\
\text { être attribuée à cette région et dont la } \\
\text { production ou la transformation ou } \\
\text { l'élaboration ont lieu dans l'aire } \\
\text { géographique délimitée. Ici, le lien avec le } \\
\text { terroir demeure à au moins un des stades de } \\
\text { la production, de la transformation ou de } \\
\text { l'élaboration et le produit peut jouir d'une } \\
\text { grande réputation »". }\end{array}$} \\
\hline \multirow{2}{*}{$\begin{array}{l}5 \text { caract. du } \\
\text { produit du } \\
\text { terroir }\end{array}$} & $\begin{array}{c}\text { Zone } \\
\text { géo. } \\
\text { limitée }\end{array}$ & $\begin{array}{l}\text { Facteurs } \\
\text { naturels }\end{array}$ & $\begin{array}{l}\text { Facteurs } \\
\text { humains }\end{array}$ & $\begin{array}{l}\text { Temps } \\
\text { tradition }\end{array}$ & $\begin{array}{c}\text { Différen- } \\
\text { ciation }\end{array}$ & $\begin{array}{c}\text { Zone } \\
\text { Geo. } \\
\text { limitée }\end{array}$ & $\begin{array}{l}\text { Facteurs } \\
\text { naturels }\end{array}$ & $\begin{array}{l}\text { Facteurs } \\
\text { humains }\end{array}$ & $\begin{array}{l}\text { Temps } \\
\text { tradition }\end{array}$ & $\begin{array}{c}\text { Différen- } \\
\text { citation }\end{array}$ \\
\hline & $\mathbf{x}$ & $\mathbf{x}$ & $\mathbf{x}$ & & $\mathbf{x}$ & $\mathbf{x}$ & & & & \\
\hline \multirow{4}{*}{ Certification } & \multicolumn{5}{|c|}{ Appellation d'origine } & \multicolumn{5}{|c|}{ Indication géographique protégée } \\
\hline & \multicolumn{5}{|c|}{ Logo } & \multicolumn{5}{|c|}{$\log ^{10}$} \\
\hline & & & & & & & & & & \\
\hline & \multicolumn{5}{|c|}{ Délivrée par le CARTV et le MAPAQ } & \multicolumn{5}{|c|}{ Délivrée par le CARTV et le MAPAQ } \\
\hline
\end{tabular}

Note : l'Agneau de Charlevoix a été officiellement reconnu le 21 mars 2009 et représente actuellement la seule IGP du Québec.

\section{CLASSIFICATION DES PRODUITS DU TERROIR ET PROFIL DES CONSOMMATEURS}

Bien que le marché des produits du terroir au Québec soit estimé à deux milliards de dollars et près d'un million et demi de consommateurs ${ }^{11}$, nous ne disposons à ce jour que de très peu d'informations pour décrire la diversité des produits constituant ce marché et mieux cerner le profil du consommateur «type» de ces produits. En effet, les recherches antérieures nous apprennent seulement que les produits du terroir au Québec ${ }^{12}$ peuvent être classés en six catégories (poissons et crustacés, fromages, boissons alcoolisées et non alcoolisées, viandes, boulangerie et pâtisseries, autres produits transformés). Par ailleurs, selon une étude réalisée en $1999^{13}$, deux variables caractérisent la relation que l'individu entretient avec les produits du 
terroir : la position socioculturelle (soit l'âge sachant que l'individu qui s'intéresse aux produits du terroir a entre 35 et 54 ans - et le sexe - plutôt féminin) ainsi que la position territoriale (zone urbaine ou rurale). Étant donné le peu de connaissances dans ce domaine, cette étude portant sur les catégories de produits agroalimentaires du terroir au Québec et sur le profil du consommateur de ce marché au Québec s'avère d'une grande actualité.

\subsection{Méthodologie de la recherche}

Dans un premier temps, nous avons recherché puis analysé des documents pertinents à notre recherche selon les critères suivants: 1) le thème central du document devait être en lien avec les produits du terroir ou un thème connexe proche (par exemple, les « appellations d'origine $»)$; 2) le document devait faire mention d'informations en lien avec les objectifs de la recherche (notamment les catégories ou typologies des produits, le profil $\mathrm{du}$ consommateur). Nous avons ainsi recensé puis étudié cent documents dont l'origine était la suivante : $29 \%$ issus des médias grand public (presse et émissions télévisées), $16 \%$ produits par les acteurs clés de la filière (production, transformation, distribution, agro-tourisme), $14 \%$ issus de centres de recherche spécialisés (en majorité universitaires), $12 \%$ provenant de la presse professionnelle du secteur (détaillants, $\mathrm{HRI}^{14}$, producteurs agricoles), $11 \%$ des ministères, organismes publics et groupes de travail en lien avec le secteur agroalimentaire, $8 \%$ de cabinets d'études ou de consultants spécialisés, $6 \%$ d'organismes de développement et de promotion du secteur (province et régions) et $4 \%$ de coalitions ou autres associations (de consommateurs ou de développement rural et agricole). Ces documents de source et de nature diverses (articles de presse, mémoires, études, rapports et articles scientifiques) ont fait l'objet d'une analyse selon une grille unique (liste des classifications possibles, commune à toutes les sources et à tous les types de documents, liste des variables possibles pour qualifier le consommateur type). Par la suite, des dénombrements ont été opérés pour identifier le nombre de fois où une classification ou une variable qualifiante était relevée dans les docu- ments à l'étude. Ce type d'analyse nous a donc permis de relever, non seulement les types de classification des produits utilisés ou les variables qualifiantes du consommateur (selon l'occurrence de l'information), mais également leur importance relative.

Dans un second temps, nous avons sollicité quatorze experts du secteur des produits agroalimentaires du terroir au Québec par le biais d'entrevues individuelles téléphoniques afin d'obtenir leur propre vision du marché (leur catégorisation et leur définition du profil type du consommateur, et ce de manière spontanée). Leurs avis et commentaires ont ensuite été recueillis sur les informations synthétisées à l'issue de l'étape précédente (divergences et convergences éventuelles). Ces entrevues d'une durée moyenne de 80 minutes ont permis d'interroger des personnes d'expérience $(86 \%$ des répondants ont plus de 10 ans d'expérience dans le secteur) issues pour l'essentiel de la production, de la transformation, de l'agrotourisme, de la distribution et du milieu des associations ou coalitions, permettant ainsi une représentation équilibrée de la diversité des acteurs intervenant dans le secteur. Une analyse par résumé a été effectuée pour faire ressortir la diversité et la richesse des points de vue des répondants.

Nous présenterons dans les paragraphes qui suivent, pour les catégories de produits et le profil type des consommateurs, les résultats principaux des deux étapes de cette recherche.

\subsection{Catégories de produits}

L'analyse documentaire fait émerger quatre modèles de référence pour la classification des produits agroalimentaires du terroir :

- selon la matière première de base du produit seulement (le bleuet, la viande d'agneau, etc.);

- selon le mode de préparation et de commercialisation (ou mise en marché) seulement (la confiture ou le pâté);

- selon la région dont est issu le produit seulement (par exemple les produits du Lac-St-Jean);

- selon un mélange de deux ou des trois classifications précédentes. 
L'analyse confirme donc l'existence dans

l'absolu de sept catégories potentielles, tel

qu'illustré à la figure 1 .

Figure 1 - Classification des produits du terroir selon 3 critères (étude documentaire)

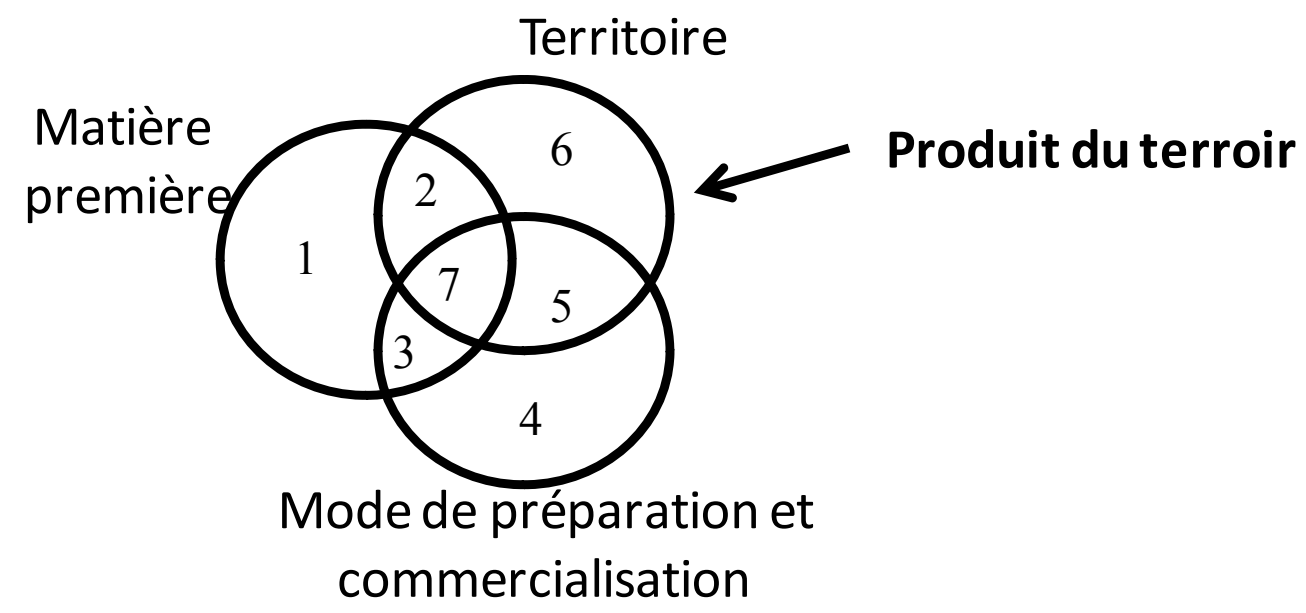

Les tableaux 2 et 3 présentent les résultats des classifications selon deux de ces trois critères (la matière première et le mode de préparation/commercialisation). La classification selon la région, très présente dans la littérature consultée, mentionne tantôt une région adminis- trative, tantôt une région touristique ou un territoire historique, parfois une zone territoriale aux contours flous, rendant ainsi la synthèse difficile et artificielle. Cette dernière classification ne sera pas dans cet article.

Tableau 2 : Classification selon la matière première

\begin{tabular}{|l|l|c|}
\hline \multicolumn{1}{|c|}{ Catégories } & \multicolumn{1}{|c|}{ Sous-catégories } & $\begin{array}{c}\text { Importance } \\
\text { relative }^{15}\end{array}$ \\
\hline Fruits & $\begin{array}{l}\text { Petits fruits et baies (sauvages ou cultivés), pommes, } \\
\text { autres fruits }\end{array}$ & $33 \%$ \\
\hline Viandes et cufs & $\begin{array}{l}\text { Volailles et gibiers à plume, agneau, chèvre et brebis, } \\
\text { animaux exotiques, boeuf, porc et veau, races anciennes } \\
\text { (ou patrimoniales), cufs, autres viandes }\end{array}$ & $33 \%$ \\
\hline Légumes & Légumes rares ou anciens, autres légumes & $13 \%$ \\
\hline Poissons et fruits de mer & Poissons, fruits de mer, autres & $11 \%$ \\
\hline $\begin{array}{l}\text { Plantes sauvages, fines herbes } \\
\text { et fleurs comestibles }\end{array}$ & $\begin{array}{l}\text { Plantes et herbes sauvages, fleurs comestibles, fines } \\
\text { herbes }\end{array}$ & $10 \%$ \\
\hline
\end{tabular}


Tableau 3 : Classification selon le mode de préparation et commercialisation

\begin{tabular}{|l|l|c|}
\hline \multicolumn{1}{|c|}{ Catégories } & \multicolumn{1}{|c|}{ Sous-catégories } & $\begin{array}{c}\text { Importance } \\
\text { relative }\end{array}$ \\
\hline Boissons alcoolisées & $\begin{array}{l}\text { Cidres, vins, bières (de microbrasserie), hydromels, autres } \\
\text { boissons alcoolisées }\end{array}$ & $23 \%$ \\
\hline Fromages et produits laitiers & Fromages, produits laitiers & $11 \%$ \\
\hline Produits de l'érable & Sirop d'érable, sucre d'érable, autres produits de l'érable & $8 \%$ \\
\hline Charcuteries & $\begin{array}{l}\text { Terrines, viandes, poissons et fruits de mer fumés, autres } \\
\text { charcuteries }\end{array}$ & $8 \%$ \\
\hline Confitures et tartinades & & $7 \%$ \\
\hline Condiments, marinades et assaisonnements & $7 \%$ \\
\hline Confiseries & Chocolats, autres confiseries & $7 \%$ \\
\hline Huiles, vinaigres et vinaigrettes & & $5 \%$ \\
\hline Produits de boulangerie & Pain (artisanal), autres produits de boulangerie & $4 \%$ \\
\hline Miels et produits de l'abeille & Miels, produits de l'abeille & $3 \%$ \\
\hline Mets préparés et plats cuisinés & Pâtés et tourtières, autres mets préparés & $3 \%$ \\
\hline Boissons non alcoolisées & Jus, eaul, thé et tisanes & $3 \%$ \\
\hline Desserts & Crèmes glacées et sorbets, autres desserts & $1 \%$ \\
\hline
\end{tabular}

De manière spontanée (sans qu'aucune catégorisation ne leur soit à priori proposée), les experts en entrevue indiquent que les produits du terroir peuvent être catégorisés comme suit :

- les grandes familles de produits agroalimentaires: fruits et légumes, viandes, produits carnés et poissons, fromages et produits laitiers, boissons et produits alcoolisés (catégories citées par $71 \%$ des experts);

- le degré de transformation : produits transformés ou non ( $21 \%$ des experts);

- le mode de fabrication : produits artisanaux et industriels ( $14 \%$ des experts);

- la région de production (14\% des experts).

Ainsi, l'analyse des entrevues révèle que, même si les experts ne mentionnent pas spontanément toutes les catégories issues de l'étude documentaire et synthétisées dans les tableaux 2 et 3, ils présentent une catégorisation similaire. En effet, leur classification par "grandes familles de produits agroalimentaires » rejoint la catégorisation selon la matière première et le mode de préparation/transformation; la classification par «degré de transformation » rejoint le mode de préparation/commercialisation et celle par « région de production", le territoire. La dichotomie «artisanal/industriel» est en revanche originale. Invités à s'exprimer sur le réalisme $^{16}$ et la pertinence ${ }^{17}$ de la classification issue de l'étude documentaire, les répondants ont majoritairement souligné qu'elle reflétait bien la réalité du marché (elle est réaliste), mais ils proposent une autre lecture (catégorisation pertinente) de la spécificité des produits du terroir au Québec (Tableau 4). 
Tableau 4 : Réalisme et pertinence des critères de classification des produits du terroir (entrevues avec les experts)

\begin{tabular}{|l|c|l|}
\hline Classification & $\begin{array}{l}\text { \%o des experts qui } \\
\text { considèrent que la } \\
\text { classification est .. }\end{array}$ & \multicolumn{1}{c|}{ Commentaires des experts } \\
\hline RÉALISTE & $79 \%$ & $\begin{array}{l}\text { Point de repère facile, très lisible et accessible pour le } \\
\text { consommateur et qui lui permet de qualifier la nature du } \\
\text { produit. }\end{array}$ \\
\hline $\begin{array}{l}\text { Matière } \\
\text { première }\end{array}$ & $71 \%$ & $\begin{array}{l}\text { La préparation représente pour le consommateur le savoir- } \\
\text { faire, l'histoire et donnent au produit du caractère, une } \\
\text { personnalité. }\end{array}$ \\
\hline $\begin{array}{l}\text { Transformation } \\
\text { ou préparation }\end{array}$ & $100 \%$ & $\begin{array}{l}\text { Le territoire est indissociable du terroir dont il facilite le } \\
\text { repérage et l'identification. }\end{array}$ \\
\hline Territoire & $7 \%$ & $\begin{array}{l}\text { La matière première fait partie du produit mais, seule, elle ne } \\
\text { permet pas de cerner ce qui rend le produit unique et } \\
\text { spécifique. }\end{array}$ \\
\hline $\begin{array}{l}\text { Matière } \\
\text { première }\end{array}$ & $14 \%$ & $\begin{array}{l}\text { La transformation ou la préparation renvoient aux notions de } \\
\text { savoir-faire humain, de culture, d'histoire et de patrimoine qui } \\
\text { sont des éléments majeurs pour les produits du terroir. }\end{array}$ \\
\hline $\begin{array}{l}\text { Transformation } \\
\text { ou préparation }\end{array}$ & $79 \%$ & $\begin{array}{l}\text { Sans territoire, on ne peut pas parler de terroir. Le territoire } \\
\text { dont il est issu fait partie intégrante du produit. }\end{array}$ \\
\hline Territoire & &
\end{tabular}

Pour eux, en effet, la référence au territoire est incontournable car le produit du terroir se définit avant tout par rapport à une zone géographique délimitée. Le territoire est donc une classification fondamentale constituant le socle de la définition et donc de la typologie des produits du terroir. Le mode de transformation/préparation serait le critère de seconde importance pour son lien avec le savoir-faire humain, la culture, l'histoire et le patrimoine qui caractérisent également les produits du terroir. Enfin pour eux, la matière première faisant partie effectivement du produit, sans toutefois en assurer la spécificité, serait le critère de troisième ordre à considérer. Ces conclusions nous amènent donc à proposer une solution alternative à la classification des produits du terroir selon les résultats combinés de l'étude documentaire et des entrevues réalisées avec les experts (Figure 2).

Figure 2 : Classification des produits du terroir selon 3 critères (étude documentaire et experts)

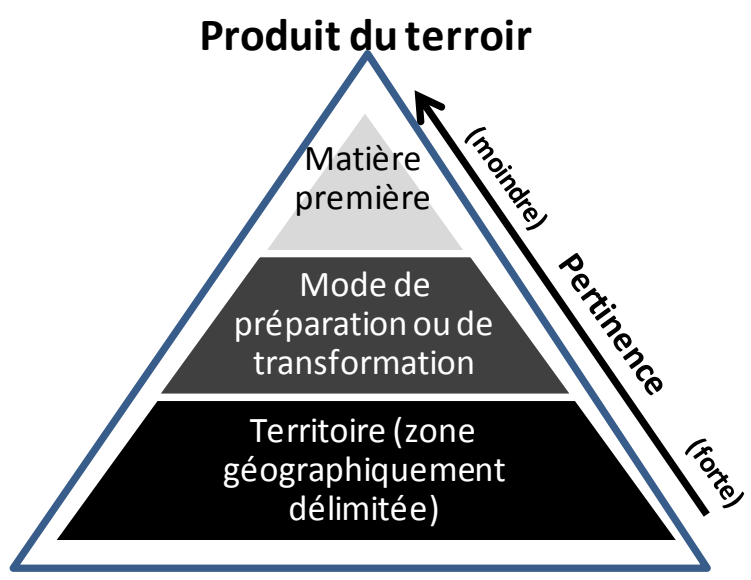




\subsection{Profil type des consommateurs}

L'analyse documentaire fait ressortir un profil type du consommateur de produits du terroir selon deux grandes familles de critères : 1) des critères comportementaux et majoritairement recensés dans les documents analysés (impor- tance relative de $83 \%$ sur l'ensemble des critères identifiés dans l'étude); 2) des critères sociodémographiques.

Ce profil type de consommateurs est présenté dans la première colonne du tableau 5 .

Tableau 5 : Profil type du consommateur de produits agroalimentaires du terroir

\begin{tabular}{|c|c|c|}
\hline $\begin{array}{l}\text { Principaux traits du profil } \\
\text { type du consommateur de } \\
\text { produits du terroir issus } \\
\text { de la revue de littérature } \\
\text { et de l'étude des données } \\
\text { secondaires } \\
\end{array}$ & $\begin{array}{l}\% \text { des } \\
\text { experts } \\
\text { qui rejoint } \\
\text { ce trait du } \\
\text { profil type }\end{array}$ & Commentaires principaux des experts \\
\hline Âge (35- 44 ans) & $21 \%$ & $\begin{array}{l}\text { Tranche d'âge considérée comme plus large : les baby-boomers et les } \\
\text { personnes plus âgées, les étudiants plus jeunes. }\end{array}$ \\
\hline Francophone & $50 \%$ & $\begin{array}{l}\text { Trait contesté. Le profil est plus lié à une culture ou une tradition } \\
\text { culinaires qu'au fait de parler une langue. }\end{array}$ \\
\hline $\begin{array}{l}\text { Loisirs... } \\
\text { Il est adepte de } \\
\text { l'agrotourisme, de } \\
\text { l'écotourisme ou du } \\
\text { tourisme culturel. }\end{array}$ & $61 \%$ & $\begin{array}{l}\text { Les experts notent que ces consommateurs sont curieux, cultivés et } \\
\text { aiment voyager. Mais, selon eux, le consommateur de produits du terroir } \\
\text { n'est pas particulièrement adepte d'écotourisme, d'agrotourisme ou de } \\
\text { tourisme culturel. }\end{array}$ \\
\hline Sexe (souvent féminin) & $71 \%$ & $\begin{array}{l}\text { Plusieurs arguments soutiennent cette affirmation : au sein du foyer, les } \\
\text { achats d'alimentation sont fréquemment féminins; sensibilité féminine } \\
\text { en phase avec la recherche de goût, de saveur et de santé liée à la } \\
\text { consommation de produits du terroir; les produits du terroir sont } \\
\text { fréquemment des achats spontanés. } \\
\text { Évolution de ce profil vers des achats et une consommation autant } \\
\text { masculins que féminins. }\end{array}$ \\
\hline Lieu de résideı & $79 \%$ & $\begin{array}{l}\text { Les consommateurs urbains recherchent via les produits du terroir un } \\
\text { lien avec la terre et le territoire, ce qu'ils n'ont plus en ville. } \\
\text { Possiblement un effet de mode. } \\
\text { Lien certain avec les lieux de distribution des produits. } \\
\text { Population urbaine fréquemment scolarisée et plus aisée que la moyenne } \\
\text { québécoise. }\end{array}$ \\
\hline $\begin{array}{l}\text { Catégorie aisée de revenus } \\
\text { (revenus supérieurs à la } \\
\text { moyenne; } 60000 \text { \$ et plus) }\end{array}$ & $79 \%$ & $\begin{array}{l}\text { Les produits du terroir sont des produits alimentaires plus chers que les } \\
\text { produits de consommation courante. } \\
\text { Ne pas négliger les consommateurs disposant de moyens financiers plus } \\
\text { limités (par exemple les étudiants) mais éduqués et intéressés par ces } \\
\text { produits. }\end{array}$ \\
\hline Travailleur à temps plein & $79 \%$ & $\begin{array}{l}\text { Profil cohérent avec le profil plutôt aisé et éduqué du consommateur type } \\
\text { de produits du terroir au Québec. }\end{array}$ \\
\hline $\begin{array}{l}\text { Scolarisé ou de niveau } \\
\text { universitaire }\end{array}$ & $93 \%$ & $\begin{array}{l}\text { Les consommateurs de produits du terroir sont cultivés, curieux et plutôt } \\
\text { intellectuels. Il semblerait plus pertinent de parler de personnes éduquées } \\
\text { et cultivées que de personnes scolarisées. }\end{array}$ \\
\hline $\begin{array}{l}\text { Comportement ... } \\
\text { Un gourmand, un gourmet, } \\
\text { un épicurien, un hédoniste; } \\
\text { Un curieux, cherchant la } \\
\text { découverte. }\end{array}$ & $93 \%$ & $\begin{array}{l}\text { Les experts soulignent la recherche effective de la nouveauté (curiosité), } \\
\text { de gourmandise et de saveur'Pour eux en revanche, la recherche de } \\
\text { plaisir seule est moins importante. }\end{array}$ \\
\hline $\begin{array}{l}\text { Préoccupations... } \\
\text { La provenance et l'origine } \\
\text { des aliments; } \\
\text { La santé et une bonne } \\
\text { alimentation; } \\
\text { L'écologie et } \\
\text { l'environnement. }\end{array}$ & $\begin{array}{l}100 \% \\
93 \% \\
86 \%\end{array}$ & $\begin{array}{l}\text { Les experts soulignent la recherche de produits dont l'origine est connue } \\
\text { (les consommateurs s'informent sur ce qu.ils mangent) et de produits } \\
\text { " santé ». Pour les experts, l'écologie et la protection de l'environnement } \\
\text { sont distincts de la recherche de produits de terroir (mais cela peut } \\
\text { intéresser des personnes de profil similaire). }\end{array}$ \\
\hline $\begin{array}{l}\text { Recherche ... } \\
\text { La qualité, l'authenticité, le } \\
\text { goût et la saveur. }\end{array}$ & $100 \%$ & $\begin{array}{l}\text { La recherche d'authenticité, de goût et de saveur prédomine selon les } \\
\text { experts par rapport à la recherche de qualité. }\end{array}$ \\
\hline
\end{tabular}


Les experts reconnaissent dans ces critères leur propre perception du marché mais en contestent également certains aspects (les $2^{\mathrm{e}} \mathrm{e}$ et $3^{\mathrm{e}}$ colonnes $\mathrm{du}$ tableau 5 présentent respectivement le pourcentage d'experts en accord avec les critères recensés et leurs commentaires principaux). Ces points de convergence et de divergence sont exposés ci-dessous.

\section{Le consommateur type recherche l'authenticité, le goût et la saveur des produits (plus encore que la qualité). Il est préoccupé par la provenance et l'origine des aliments et leur profil « santé ». Il est gourmand, gourmet, épicurien, hédoniste et curieux, et cherche à expérimenter des produits nouveaux.}

Selon les experts, le consommateur type recherche l'authenticité, le goût et la saveur des produits (plus encore que la qualité). Il est préoccupé par la provenance et l'origine des aliments et leur profil « santé ». Il est gourmand, gourmet, épicurien, hédoniste et curieux, et cherche à expérimenter des produits nouveaux. Le consommateur type est très éduqué et cultivé, urbain et plutôt aisé (car ces produits sont perçus comme étant plus chers que les produits agroalimentaires usuels) et donc travailleur à temps plein.

En revanche, la préoccupation écologiste, la protection de l'environnement et, dans une moindre mesure, l'agrotourisme ne sont pas des facteurs aussi déterminants dans son profil que les précédents. Les experts nuancent également l'affirmation selon laquelle le consommateur serait plutôt «une consommatrice» (ils constatent une évolution vers une répartition autant masculine que féminine) et qu'il serait âgé de 35 à 44 ans (ils pensent que cette tranche d'âge devrait absolument être élargie aux «baby-boomers" plus vieux et aux clientèles plus jeunes comme les étudiants). Ils contestent également l'affirmation selon laquelle il serait seulement francophone.

La vision spontanée des experts quant au consommateur type de produits du terroir est exposée au tableau 6. Elle est cohérente avec leurs critiques et commentaires exposés précédemment.

\section{Tableau 6 : Profil type du consommateur des produits du terroir au Québec (vision spontanée des experts interrogés)}

\begin{tabular}{|l|c|}
\hline \multicolumn{1}{|c|}{$\begin{array}{c}\text { Principaux traits du profil type } \\
\text { du consommateur de produits du terroir au Québec }\end{array}$} & $\begin{array}{c}\text { \% des experts } \\
\text { ayant proposé } \\
\text { cette } \\
\text { classification }\end{array}$ \\
\hline $\begin{array}{l}\text { Personnalité de l'individu consommateur (centres d'intérêt) : cultivé, ouvert d'esprit et } \\
\text { intéressé par des cultures diverses différentes de la sienne; curieux, cherchant la } \\
\text { découverte et de nouvelles expériences; informé et faisant des choix éclairés; ayant } \\
\text { voyagé et intéressé par les voyages; gourmand, gourmet; épicurien et hédoniste; } \\
\text { convivial. }\end{array}$ & $\mathbf{7 1 \%}$ \\
\hline $\begin{array}{l}\text { Situation financière de l'individu consommateur : financièrement à l'aise; revenus } \\
\text { décents. }\end{array}$ & $\mathbf{5 7 \%}$ \\
\hline $\begin{array}{l}\text { Attentes du consommateur vis-à-vis des produits : qualité; authenticité, véracité des } \\
\text { produits; originalité et unicité des produits; goût et saveur. }\end{array}$ & $\mathbf{5 0 \%}$ \\
\hline $\begin{array}{l}\text { Niveau d'éducation ou de formation du consommateur : scolarisé (scolarisation } \\
\text { postsecondaire ou universitaire, scolarisation supérieure à la moyenne); éduqué; instruit } \\
\text { (culture générale). }\end{array}$ & $\mathbf{5 0 \%}$ \\
\hline $\begin{array}{l}\text { Motivations et préoccupations du consommateur : santé; alimentation saine et } \\
\text { équilibrée; provenance ou origine des produits; procédé de fabrication et de } \\
\text { transformation des produits; écologie. }\end{array}$ & $\mathbf{3 6 \%}$ \\
\hline $\begin{array}{l}\text { Âge de l'individu consommateur : «baby-boomers » (tranche des } 45-65 \text { ans); jeunes et } \\
\text { jeunes professionnels (générations X et Y). }\end{array}$ & $\mathbf{2 1 \%}$ \\
\hline
\end{tabular}




\section{CONCLUSION}

\section{Cette recherche exploratoire a permis de mieux cerner la définition des produits agroalimentaires du terroir au Québec, leurs caractéristiques distinctives, les classifications possibles et le profil type du consommateur de ces produits.}

Cette recherche exploratoire a permis de mieux cerner la définition des produits agroalimentaires du terroir au Québec, leurs caractéristiques distinctives, les classifications possibles et le profil type du consommateur de ces produits. Les connaissances aussi élaborées sur les catégories de produits, du profil comportemental et sociodémographique des consommateurs permettront sans aucun doute de réaliser d'autres recherches avec des bases plus solides. Par exemple, ces données nous permettront de construire, soit des plans d'expérience pertinents (par exemple selon toutes les catégories de produits du terroir représentatives du marché dans son ensemble), soit des échantillons représentatifs des consommateurs de ces produits (par exemple selon une méthode d'échantillonnage empirique, selon la méthode des quotas).

Toutefois, l'approche utilisée (étude documentaire et qualitative) limite la généralisation des données obtenues. Les résultats représentent bien la réalité du marché (car ils ont été recensés dans la documentation et cités par les experts), mais l'approche utilisée ne permet pas de conclure définitivement sur leur importance relative. Il conviendra donc, dans des recherches futures quantitatives (par questionnaire auprès d'un échantillon représentatif de la population québécoise) de mesurer par exemple les variables comportementales et sociodémographiques relevées.

Le projet de recherche à l'origine de cette étude exploratoire $^{4}$, sur la base de ces données, s'intéressera désormais à la perception par les consommateurs des produits du terroir au Québec. Notamment, en référence au concept de «valeur perçue » (« une évaluation globale par le consommateur de l'utilité d'un produit basée sur les perceptions de ce qui est reçu et de ce qui est donné ${ }^{18}$ ), il tentera d'établir quantitativement la nature et l'importance relative des bénéfices perçus (liés à la qualité du produit, à la satisfaction personnelle ressentie de contribuer au développement de sa communauté, par exemple) recherchés dans l'achat et la consommation de ces produits. Il pourra également étudier les effets de l'apposition d'une certification au produit du terroir (par exemple un label) sur cette perception. L'enrichissement de la connaissance relative à ce marché permettra aux producteurs de mieux valoriser leurs produits et donc de contribuer effectivement au développement de l'économie des terroirs.

\section{BIBLIOGRAPHIE}

Les auteures adressent leurs remerciements aux experts qui, au Québec, ont bien voulu consacrer du temps à la recherche et répondre aux questions qui leur étaient soumises sur les produits du terroir. Elles sont également reconnaissantes au Fonds Québécois de Recherche sur la Société et la Culture (FQRSC) qui a permis de réaliser les recherches à la base de cette publication (la professeure Catherine Parissier a obtenu en 2008 un financement de trois ans, dans le cadre du programme Établissement de nouveaux professeurs-chercheurs, sur le thème de la Valeur perçue des produits agroalimentaires du terroir au Québec). Elles remercient également les lecteurs anonymes et la Direction de la Revue pour leurs commentaires pertinents ayant permis la rédaction de la version finale de cet article.

${ }^{1}$ Par l'entremise, par exemple, d'émissions télévisées culinaires telles que « Des kiwis et des hommes» ou «L'épicerie » diffusées en juillet 2010 à la première chaine de Radio Canada.

${ }^{2}$ Par le biais, par exemple, de sites Internet tels que http://www.terroirsquebec.com/ ou

http://www.alimentsduquebec.com/index.php (consultés en juillet 2010).

${ }^{3}$ Turgeon I. et Parissier C. (2007). «Les types de valeur perçue des produits du terroir dans un contexte québécois », Organisations et Territoires, 16, 2 et 3, 26-38.

${ }^{4}$ Projet de recherche «La valeur perçue des produits agroalimentaires du terroir au Québec »; Subvention 2007-2010 du Fonds de Recherche sur la Société et la Culture (FQRSC); Programme «Établissement de nouveaux professeurs-chercheurs $»$. 
${ }^{5}$ Gélinas N. (2002). «De nature à culture; les produits du terroir; premier inventaire des produits du terroir québécois », Collection Études Rurales, Solidarité Rurale du Québec, Nicolet Québec Éditeur.

${ }^{6}$ Le CARTV est chargé depuis 2006 par le Gouvernement du Québec de l'application de la loi de 2006 sur les appellations réservées et les termes valorisants au moyen d'un processus de certification.

${ }^{7}$ Jungbauer, M. (2010). «L'influence d'un label sur la valeur perçue des produits du terroir québécois », Mémoire de recherche en vue de l'obtention du grade de Maitre ès Sciences, Faculté d'administration de l'Université de Sherbrooke (Qc), Canada.

${ }^{8} \mathrm{http}: / /$ www.cartvquebec.com/fr/appellationdorigine, consulté en mai 2010.

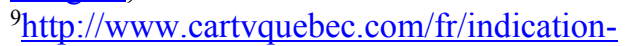
geographique-protegee, consulté en mai 2010.

${ }^{10}$ Note : l'Agneau de Charlevoix a été officiellement reconnu le 21 mars 2009 et représente actuellement la seule IGP du Québec.

${ }^{11}$ Conférence de Madame Nathalie Normandeau, ministre déléguée au développement régional et au tourisme, $13^{\mathrm{e}}$ conférence de Solidarité Rurale du Québec, 2005.

${ }^{12}$ Ibid 5.

${ }^{13}$ « Étude des comportements et attitudes des Québécois à l'égard de la ruralité et des produits du terroir », projet 9115, juin 1999, Jolicoeur \& Associés, p. 32-33.

${ }^{14}$ Hôtels, Restaurants et Institutions.

${ }^{15}$ L'importance relative de la catégorie " $\mathrm{x}$ 》 a été calculée comme suit : nombre de fois où la catégorie $\mathrm{x}$ a été relevée dans les documents étudiés / nombre total de citations de toutes les catégories confondues relevées dans les documents étudiés.

${ }^{16}$ Afin de juger du réalisme de la classification proposée, les répondants ont été invités à s'exprimer sur le fait que cette dernière reflétait, selon leurs connaissances et leur jugement personnel, bien, partiellement ou inadéquatement la réalité québécoise des produits agroalimentaires du terroir québécois. ${ }^{17} \mathrm{Afin}$ de juger de la pertinence de la classification proposée, les répondants ont été invités à s'exprimer sur le fait que cette dernière permettait de disposer d'une lecture bien, partiellement ou inadéquatement adaptée à la spécificité des produits du terroir québécois.

${ }^{18}$ Zeithaml V.A. (1988). Consumer Perceptions of Price, Quality and Value: A Means-End Model and Synthesis of Evidence, Journal of Marketing, 52, 2-22. 


\section{Les études de cycles supérieurs à I'UQAC}

ARTS ET LETTRES · Maîtrise en art · Maîtrise en lettres · Maîtrise en linguistique $\cdot$ Doctorat en lettres

SCIENCES HUMAINES - Programme court en études régionales - Maîtrise en études et interventions régionales $\cdot$ Doctorat en développement régional $\cdot$ Diplôme en sciences infimières $\cdot$ Maîtrise en sciences infirmières • Maîtrise en médecine expérimentale $\cdot$ Maîtrise en travail social $\cdot$ Programme court d'accompagnement des aînés · Programme court en éthique $\cdot$ Programme court en études religieuses

SCIENCES DE L'ÉDUCATION ET DE PSYCHOLOGIE • Diplôme en intervention éducative • Diplôme en enseignement collégial • Maîtrise en éducation • Doctorat en psychologie profil intervention • Doctorat en éducation Programme court en intervention éducative $\cdot$ Programme court en administration scolaire • Diplôme en administration scolaire • Diplôme en orthopédagogie

SCIENCES ÉCONOMIQUES ET ADMINISTRATIVES - Programme court en gestion de projet

- Diplôme en gestion de projet $\cdot$ Maîtrise en gestion de projet $\cdot$ Maîtrise en gestion des organisations - Maîtrise en administration des affaires (MBA) • Diplôme en sciences comptables

SCIENCES APPLIQUÉES • Maîtrise en ingénierie • Doctorat en ingénierie • Diplôme en sciences de la Terre $\cdot$ Maîtrise en sciences de la Terre $\cdot$ Doctorat en ressources minérales

SCIENCES FONDAMENTALES - Maîtrise en ressources renouvelables · Doctorat en sciences de l'environnement • Doctorat en biologie $\cdot$ Programme court en écoconseil $\cdot$ Diplôme en éco-conseil • Programme court de deuxième cycle en gestion durable du carbone forestier

INFORMATIQUE ET MATHÉMATIQUE

- Diplôme en informatique appliquée $\cdot$ Maîtrise en informatique $\cdot$ Doctorat en sciences et technologies de l'information

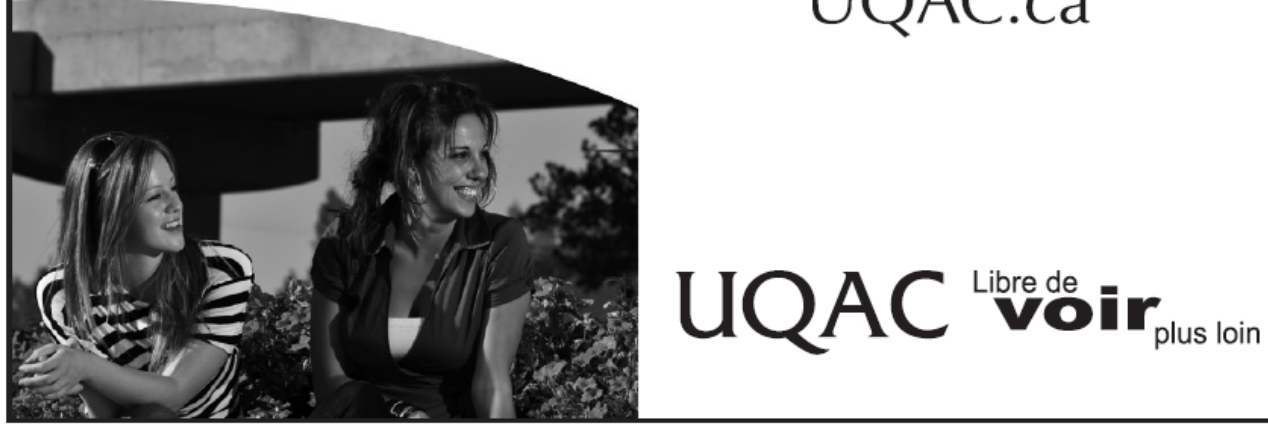

\section{Consultez nos programmes UQAC.ca}

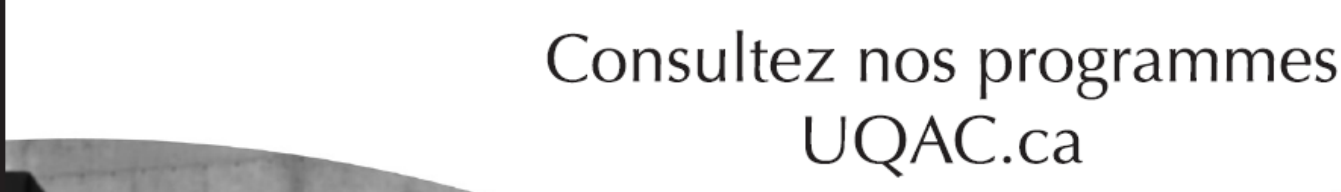

